\title{
INVESTIGATING PROPAGATION CURVES OF ELECTRIC FIELD INTENSITY OF JIGAWA MEDIUM FREQUENCY RADIO TRANSMITTER
}

\author{
R. A. Adenodi* \\ Department of Physics, Adeyemi College of EducAtion, Ondo, ONdo StATE, NIGERIA \\ E-mail address: adenodiraph@gmail.com
}

\begin{abstract}
This study investigates the variations in the electric field intensity of Jigawa Medium Frequency (MF) radio signal along some radio routes using propagation curve. It also compares the propagation curves due to measured field intensity with those predicted by GRWAVE software using the ground electrical conductivity of the routes. The need to predict propagation curves using GRWAVE software and compare same with empirical curves to ascertain the adequacy and suitability of the predicted curves to characterize MF propagation in Northern Nigeria is the motivation for this study. Electric field intensity data were acquired with the aid of Potomac electric field intensity meter and a Garmin 38Global Position System (GPS) receiver registered both coordinates and elevations along the routes. Theoretical equation was used to deduce the values of ground electrical conductivity from the measured field intensity and the values were used along with the transmitter configuration in GRWAVE software to compute the predicted field intensity. Results reveal experimental propagation curves are fairly higher compared to predicted curves. At $30 \mathrm{~km}$ from the transmitter, the values of measured and predicted electric field strength are 1.30 and $1.06 \mathrm{mV} / \mathrm{m}$ along Tundun Wada route, 1.50 and $1.11 \mathrm{mV} / \mathrm{m}$ along Azare route, 1.40 and 1.15 $\mathrm{mV} / \mathrm{m}$ along Kefin Hausa route, 1.41 and $1.13 \mathrm{mV} / \mathrm{m}$ along Kogin Maikaho route, 1.26 and 1.03 $\mathrm{mV} / \mathrm{m}$ along Kundun route, 1.50 and $1.11 \mathrm{mV} / \mathrm{m}$ along Fulani route, and 1.25 and $1.11 \mathrm{mV} / \mathrm{m}$ along Dutse Kan route. Therefore GRWAVE software can be used to predict propagation curves suitable for MF propagation in Northern Nigeria using the ground electrical conductivity of the terrain.
\end{abstract}

Key word: Propagation curve, electric field intensity, ground electrical conductivity, ground wave.

\section{INTRODUCTION}

The medium frequency (MF) lies between 300 and $3000 \mathrm{kHz}$. Services available on this frequency band includes; mobile, navigation and broadcasting [1]. Radio broadcasting is undertaken in the frequency range $525-1705 \mathrm{kHz}$ in the medium wave band. The MF amplitude modulated band had been one of the most used broadcasting techniques for decades, allowing information to arrive at innumerable listeners within localities. It exhibits covert coverage, which ensures no skip zone [2]. Wider coverage and better audio quality attract increased listeners' interest and more patronage. Many analogue MF broadcasting systems have been changed to digital rather than do away with the system in the face of many alternatives. Propagation at MF band is by sky and ground waves [1]. Ground waves follow the curvature of the earth while sky wave propagates by reflection from the ionosphere. Sky wave is absorbed during the day because of increased ionization and therefore ground wave dominates [3].

Electric field intensity at this frequency band is not sensitive to permittivity changes but fully dependent on conductivity, transmitter power, polarization, transmitter and receiver antenna heights, receiver's distance from transmitter, operating frequency,

* Corresponding author, tel: $+234-803-442-5682$ 
season, absorption by vegetation and other obstructing features along the signal paths [4-8].

Radio signals radiated by antenna are made of electric and magnetic field components. The electric field component is measured as change of potential over a given distance and this is known as the field intensity $E$ in volts per meter $[9,10]$. The electric field intensity at a distance $d(\mathrm{~km})$ from the transmitter is given as [11];

$$
E=\frac{300 A \sqrt{P}}{d}
$$

where $P$ is the effective radiated power in $\mathrm{kW}$ and $A$ is the ground attenuation function. The attenuation function in a complicated way depends on operating frequency $f$, the distance from the transmitter, permittivity $\varepsilon$ and conductivity $\sigma$ of the ground [12]. However, the function is expressed in terms of the phase constant $b$ and the numerical distances [11].

$$
A=e^{-0.43 s+0.01 s^{2}}-\sin b \sqrt{\frac{s}{2}} \exp \left(-\frac{5 s}{8}\right)
$$

when $s \leq 4.5$ and

$$
A=\frac{1}{2 s-3.7}-\sin b \sqrt{\frac{s}{2}} \exp \left(-\frac{5 s}{8}\right)
$$

when $s>4.5$

The computation for $s$ and $b$ depends on the polarization of the signals. For vertical polarization:

$$
\begin{aligned}
& s=\frac{\pi d f^{2} \cos b}{54 \times 10^{2} \sigma} \\
& b=\tan ^{-1}\left[\frac{(\varepsilon+1) f}{18 \times 10^{3} \sigma}\right]
\end{aligned}
$$

For horizontal polarization:

$$
\begin{gathered}
s=\frac{6 \times 10^{4} \sigma \pi d}{\cos b} \\
b=\tan ^{-1}\left[\frac{(\varepsilon-1) f}{18 \times 10^{3} \sigma}\right]
\end{gathered}
$$

Horizontal polarization is not usually used because it signals are always attenuated within a short distance. A plot of electric field intensity $E$ against distance $d$ yields propagation curve. Propagation curve is the variation of theelectric field intensity of the radio signals with distance $[13,14]$. It represents the reduction in electric field intensity along the propagation path. An understanding of the propagation characteristics over the terrain where the transmitter is licensed to cover is essential for predicting the system performance, radio propagation planning and designing $[15,16]$.

Among the ground electrical parameters, only ground electrical conductivity influences radio wave propagation at the MF band [5]. If the conductivity is high the attenuation will be less. The depth of penetration of the surface wave is inversely proportional to the frequency and the ground electrical conductivity. The energy of the wave is channeled within a region located just above the surface; little energy penetrates deeper into the ground. This effect allows the signal to be detected at large distances. In contrast, when the ground electrical conductivity is low, wave penetrates deeper into the ground and the distance from the transmitter at which the signals can be detected is less than that associated with high conducting medium $[17,18]$.

Another factor that can cause variation in the electric field intensity as the wave propagates is absorption by vegetation [19]. An increase in vegetation height results in an increase in signal absorption. The data for this study was collected during dry season when vegetation height is generally at its lowest in Jigawa State. Therefore signals variation due to absorption by vegetation is considered minimum and hence, ground electrical conductivity and distance are considered the major reason for signals variation in this study. In wet season, absorption by vegetation will increase thus reducing electric field strength but conductivity will increase due to ground wetness thereby enhancing electric field strength.

The motivation for this study is the need to predict propagation curves using GRWAVE software and compare same with experimental curves to ascertain the adequacy and suitability of the predicted curves to characterize MF propagation in Northern Nigeria. The sources of information on propagation curves were those contained in the Recommendations of International Telecommunication Union [15, 20] and the Radio Wave Propagation Research Group of Obafemi Awolowo University [13]. The main limitation of the curves from these sources is that they are produced on the assumption that the ground electrical characteristics were uniform over the whole path. This study involves radio wave propagation over terrain with mixed electrical characteristics.

\section{MATERIAL AND METHODS}

Measurement campaign of electric field intensity took place along seven different routes with the aid of Potomac field intensity meter and a Garmin 38 GPS receiver. The interval between two consecutive points of measurement ranges between 5 and $10 \mathrm{~km}$ depending on the availability of space to stop the vehicle used for the test drive, which took place between the hours of 9 am and $5 \mathrm{pm}$ so as to avoid measuring the electric field intensity of space wave. 
The values of measured electric field intensity were plotted against the distance for each route to produce empirical propagation curve. Equations $1-5$ were used to deduce the ground electrical parameters from the measured electric field intensity. The deduced electrical parameters together with the transmitter configuration were imputed into GRWAVE software to theoretically predict the electric field intensity, which were later used to generate the predicted propagation curves.

GRWAVE software is an ITU-R ground wave MS DOS software developed in FORTRAN to estimate the electric field intensity produced at points with known ground electrical characteristics and antennae heights $[5,6,19,21,22]$. Input parameters are the heights of transmitting and receiving antennas, polarization of transmitting antenna, frequency and ground electrical conductivity of the terrain. In this study, the software was used to estimate the electric field intensity of the signal of Jigawa Radio station. Transmitting antenna heights and operating frequencies were obtained from the broadcasting station. The receiving antenna height was maintained at $2.50 \mathrm{~m}$ throughout the measurement campaign while the polarizations are usually vertical. The transmitter configuration is presented in Table 1.

Table 1: Configuration of Jigawa Radio Transmitter

\begin{tabular}{ll}
\hline Transmitter Power & $25 \mathrm{~kW}$ \\
Frequency & $1026 \mathrm{kHz}$ \\
Height of Mast & $182.88 \mathrm{~m}$ \\
& Latitude $11^{\circ} 42^{\prime} 32.30^{\prime \prime} \mathrm{N}$ \\
Coordinates of Mast & $\begin{array}{l}\text { Longitude } 9^{\circ} 21^{\prime} 6.40^{\prime \prime} \mathrm{E} \\
\text { Altitude } 720.0 \mathrm{~m}\end{array}$ \\
Name of Location & Dutse, Dutse LGA Jigawa \\
Licensed Area & Landmass of Jigawa State \\
\hline
\end{tabular}

The experimental propagation curves for all the routes were compared to deduced variations in terms of distance covered and electric field intensity level. Similar procedures were carried out on the predicted propagation curves and the terrain profiles. The experimental curve for each route was compared with predicted curve against the path profile to deduce variations in the electric field intensity level against altitude. Logarithm scale was used on the vertical axes of the curves because the values of the signals in $m V / m$ are small compared to their equivalent in $d B \mu V / m$. On the horizontal axis, electric field intensity started close to zero mark because measurement began at $1.0 \mathrm{~km}$ away from the transmitter.

\section{RESULTS AND DISCUSSION}

The experimental curves and predicted curves are separately compared in Figures 1 and 2 respectively. The difference between the electric field intensity levels of the experimental curves for a range of $1.0 \mathrm{~km}$ to about8.0 $\mathrm{km}$ is not obvious because the electric field intensity at the distance is uniformly strong in all directions but it reduced with distance and the effect of variations in altitude and ground electrical conductivity is of no significant. Variations in the curves begin at a distance of about $10.0 \mathrm{~km}$ and continue to about above $90.0 \mathrm{~km}$. This is evident in Figures 1 and 2 as the curves spread out after $10 \mathrm{~km}$ distance from the transmitter.

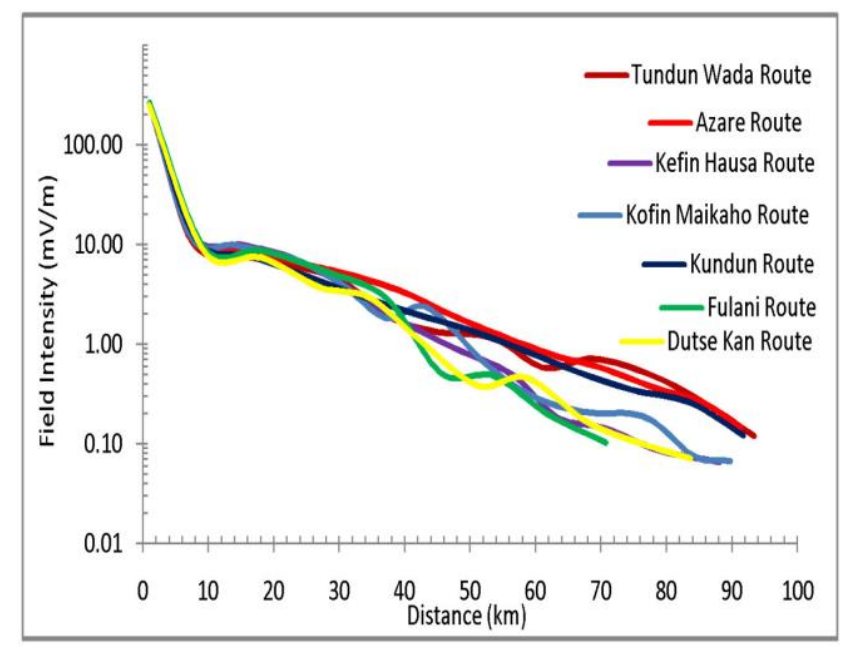

Figure 1: Experimental Propagation Curves of Jigawa Radio Transmitter

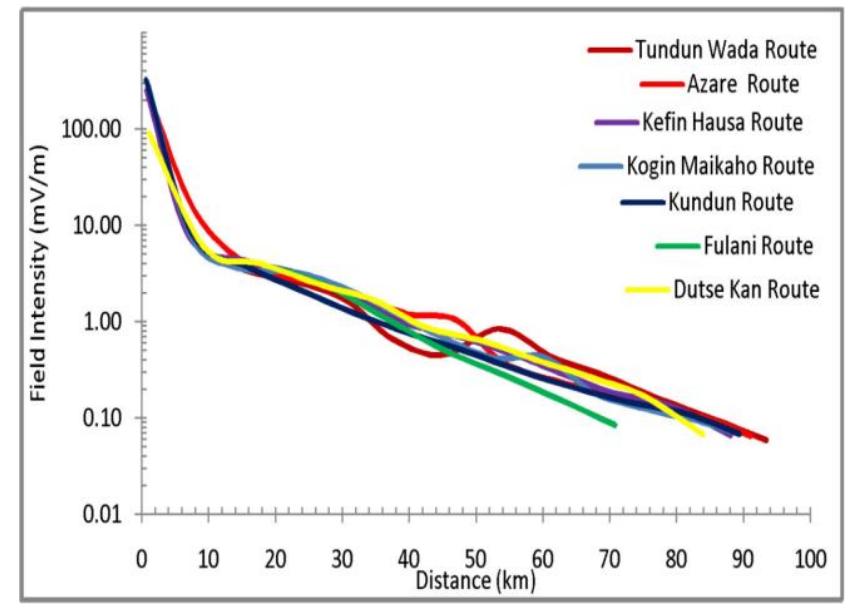

Figure 2: Theoretical Propagation Curves of Jigawa Radio Transmitter

There is a sharp reduction in the value of the electric field intensity from about $102 \mathrm{mV} / \mathrm{m}$ at a distance $1.0 \mathrm{~km}$ to about $10 \mathrm{mV} / \mathrm{m}$ at a distance of $8.0 \mathrm{~km}$. 
This is due to free space loss caused by spreading out of the signal. There is a noticeable variation in the predicted propagation curves in the $1.0 \mathrm{~km}$ to $8.0 \mathrm{~km}$ range. While the curve for Dutse Kan route began at $100 \mathrm{mV} / \mathrm{m}$, the others started at a little above $102 \mathrm{mV} / \mathrm{m}$. Similarly, the curve for Azare route has a value of $11 \mathrm{mV} / \mathrm{m}$ while others have a value of $10 \mathrm{mV} / \mathrm{mat}$ of $8.0 \mathrm{~km}$.

At $45.0 \mathrm{~km}$, the experimental propagation curve for Fulani route has the lowest value of $0.3 \mathrm{mV} / \mathrm{m}$ while that of Azare route has the highest value of $1.1 \mathrm{mV} / \mathrm{m}$. On the predicted propagation curve, Tundun Wada has the lowest electric field intensity of $0.4 \mathrm{mV} / \mathrm{m}$ while Azare has the highest value of $1.0 \mathrm{mV} / \mathrm{m}$. At $80 \mathrm{~km}$, Kefin curve has a value of $0.07 \mathrm{mV} / \mathrm{m}$ while Tundun Wada has $0.4 \mathrm{mV} / \mathrm{m}$ on the experimental curves. On the predicted curves, Dutse Kan has $0.1 \mathrm{mV} / \mathrm{m}$ while Tundun Wada has $0.2 \mathrm{mV} / \mathrm{m}$. In each case, the curves spread out as the distance from the transmitter increases. This implies increased variation in electric field intensity as distance increases.

The variation in altitude is not pronounced as presented in Figure 3. The surface of the landmass is fairly plain as the difference in maximum and minimum altitudes recorded over an area of landmass with about $95 \mathrm{~km}$ in radius is $150 \mathrm{~m}$. The transmitter is positioned at an altitude of $720.0 \mathrm{~m}$ which is the highest within the coverage area. Tundun Wada and Dutse Kan routes have their maximum at altitude of $670 \mathrm{~m}$ at $78 \mathrm{~km}$ and $48 \mathrm{~km}$ respectively while Kefin Hausa and Azare routes have their minimum altitude of $520 \mathrm{~m}$ at $21 \mathrm{~km}$ and $43 \mathrm{~km}$ respectively.

Generally, experimental curves have higher values when compared to predicted curves in all the routes except at some few distances along Kefin Hausa, Kogin Maikaho and Dutse Kan routes. Along Tundun Wada route, both curves have the same value of electric filed intensity for the first $5 \mathrm{~km}$ but after that the experiment curve became higher than the predicted curve. As shown in Figure 4, the separation widen until it reaches about $0.6 \mathrm{mV} / \mathrm{m}$ at $41 \mathrm{~km}$ and then reduced to about $0.2 \mathrm{mV} / \mathrm{m}$ at $56 \mathrm{~km}$. It later widens to $0.7 \mathrm{mV} / \mathrm{m}$ at $74 \mathrm{~km}$ and end at $0.22 \mathrm{mV} / \mathrm{m}$. The path profile is on the average smooth for the wave to follow its contour. Along Azare route, both curves have the same value of electric filed intensity for the first $10 \mathrm{~km}$ but thereafter the experiment curve became higher than the predicted curve. As shown in Figure 5, the difference varied between $4.0 \mathrm{mV} / \mathrm{m}$ at $26 \mathrm{~km}, 2.0 \mathrm{mV} / \mathrm{m}$ at $49 \mathrm{~km}$ and $0.3 \mathrm{mV} / \mathrm{m}$ at $80 \mathrm{~km}$.
The path profile is fairly smooth for the wave to follow its contour.

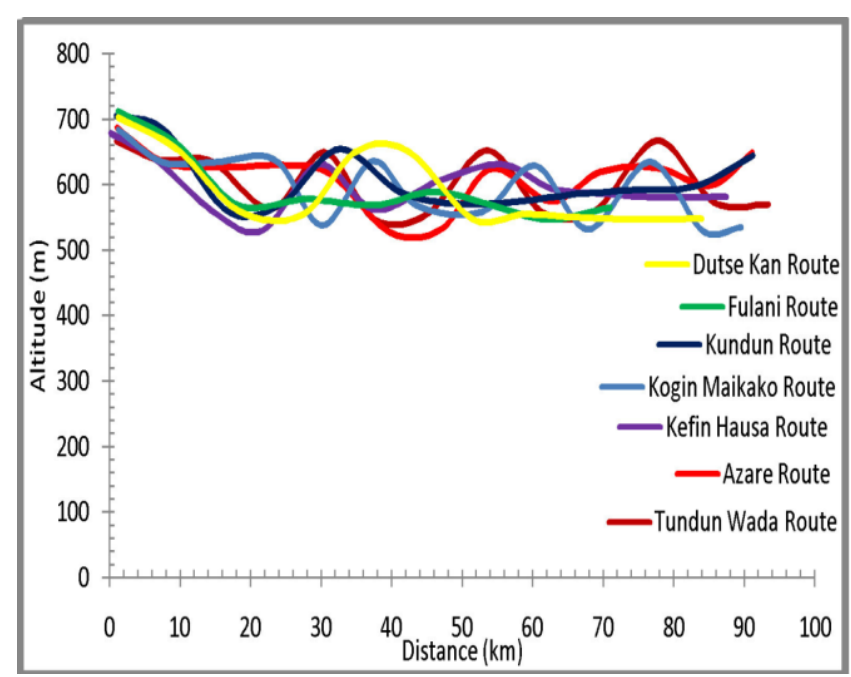

Figure 3: Path Profiles of Radio Routes

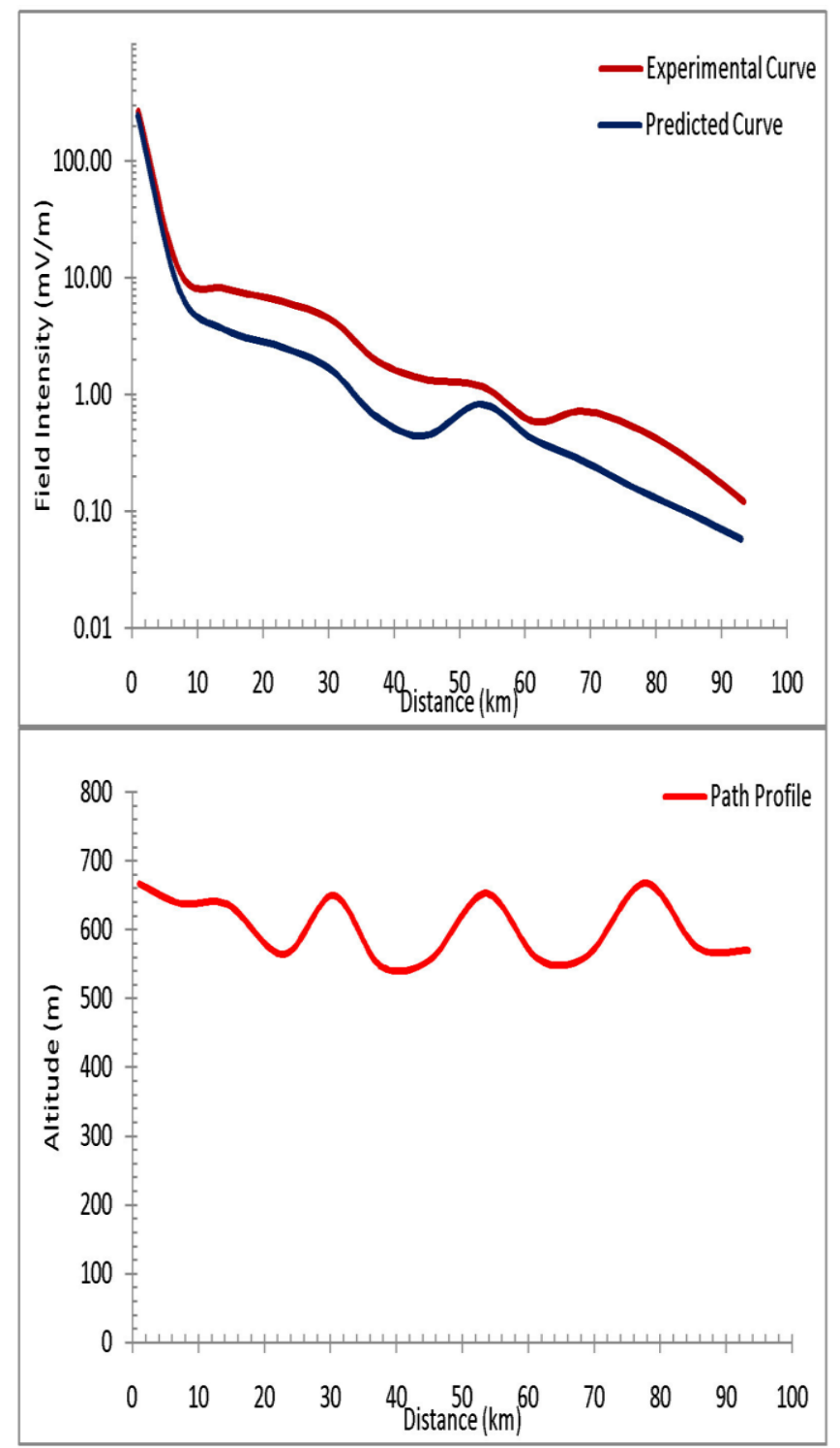

Figure 4: Propagation Curve and Terrain Profiles along Transmitter - Tundun Wada Route 


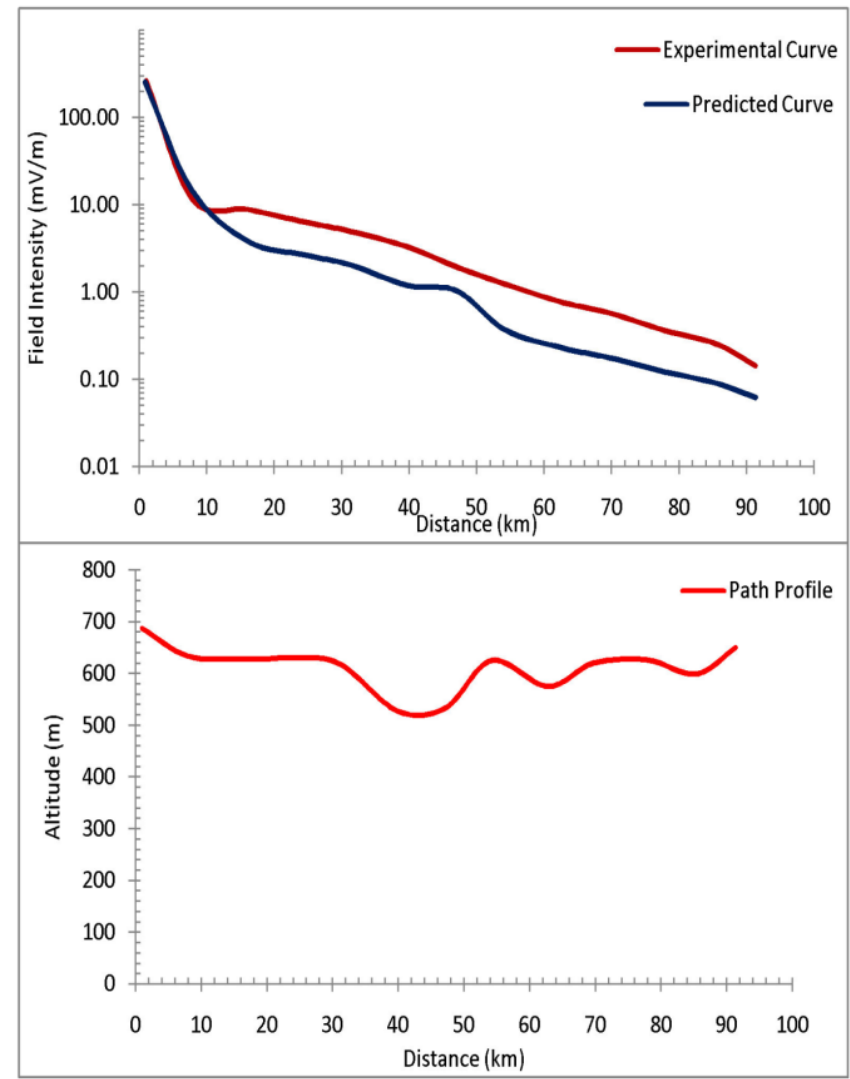

Figure 5: Propagation Curve and Terrain Profiles along Transmitter - Azare Route
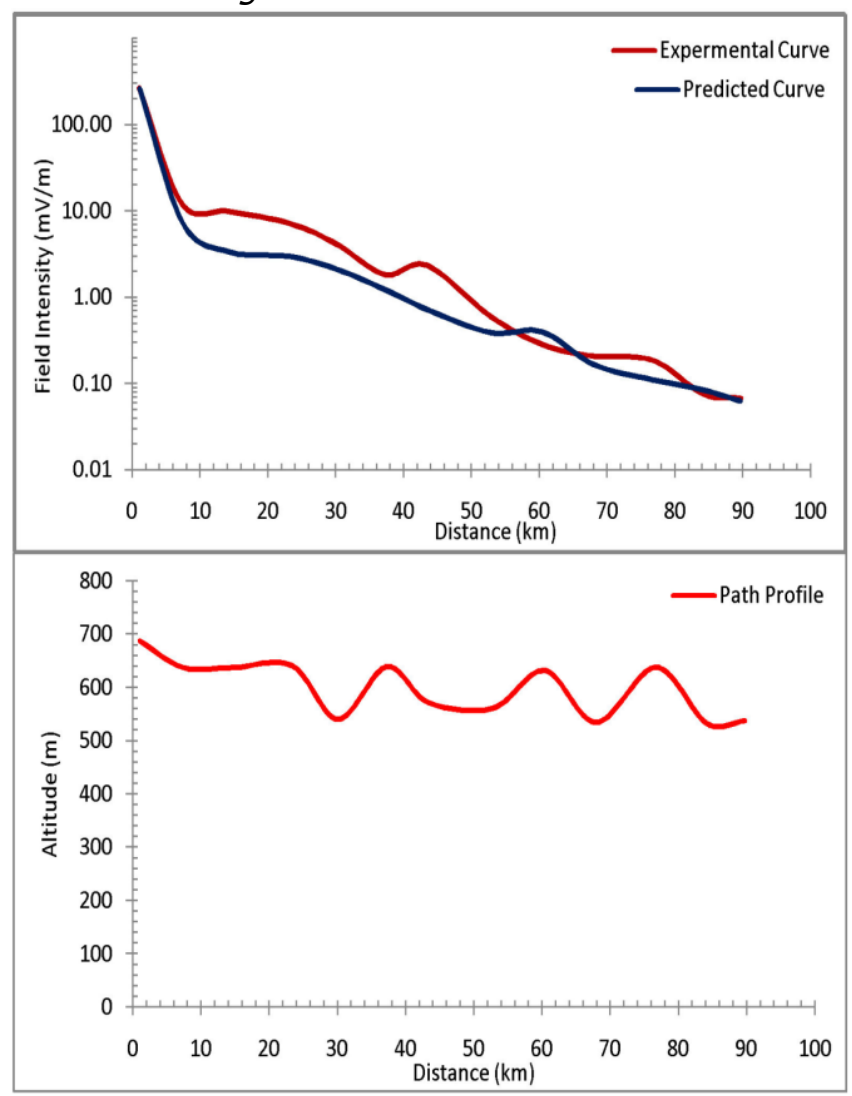

Figure 7: Propagation Curve and Terrain Profiles along Transmitter - Kogin Maikaho Route
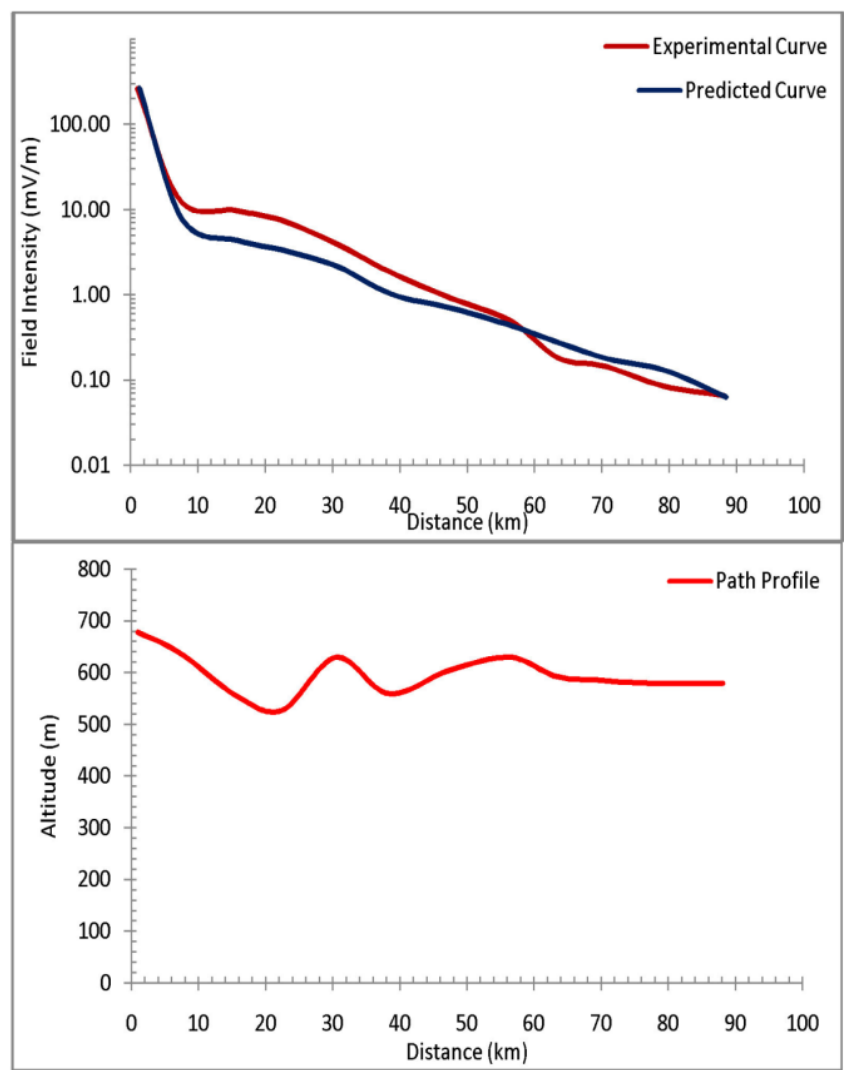

Figure 6: Propagation Curve and Terrain Profiles along Transmitter - Kefin Hausa Route
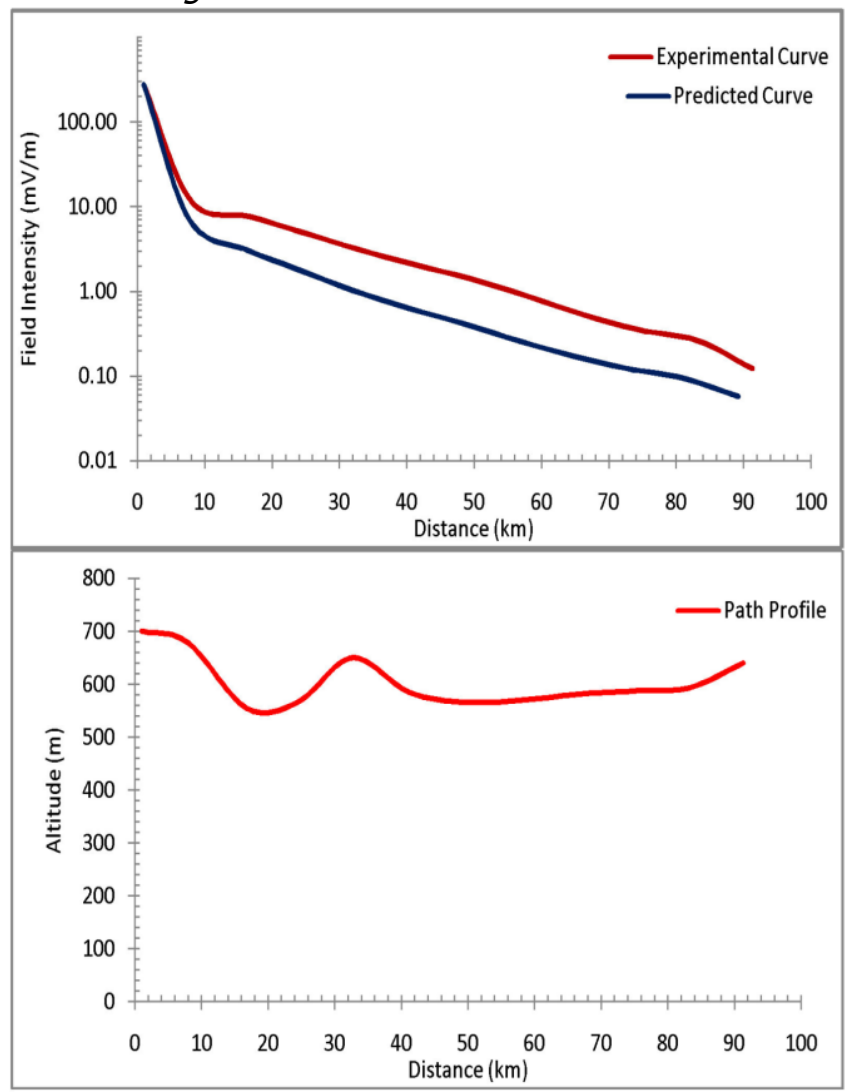

Figure 8: Propagation Curve and Terrain Profiles along Transmitter - Kundun Route 
The curves have the same value of electric filed intensity for the first $5 \mathrm{~km}$ along Kefin Hausa route as shown in Figure 6. Experimental curve remains higher until predicted curve took over at $59 \mathrm{~km}$ and remain higher until they have the same value again at $89 \mathrm{~km}$.Experimental curve was higher by about $4.0 \mathrm{mV} / \mathrm{m}$ at $20 \mathrm{~km}$ while predicted curve was higher by about $1.0 \mathrm{mV} / \mathrm{m}$ at $80 \mathrm{~km}$. The path profile is smooth for the wave to follow its contour. Both curves have the same value of electric filed intensity for the first $6 \mathrm{~km}$ along Kogin Maikaho route as shown in Figure 7. Experiment curve remains higher until predicted curve took over at $57 \mathrm{~km}$ and experimental curve became higher again at $66 \mathrm{~km}$ until $84 \mathrm{~km}$ when both curves have almost the same value. Experimental curve was higher by $6.0 \mathrm{mV} / \mathrm{m}, 2.1 \mathrm{mV} /$ $m$ and $3.0 \mathrm{mV} / \mathrm{m}$ at $20 \mathrm{~km}, 43 \mathrm{~km}$ and $75 \mathrm{~km}$ respectively. Predicted curve is higher by $0.15 \mathrm{mV} / \mathrm{m}$ at $61 \mathrm{~km}$.The path profile is considerably smooth for the wave to follow its contour. Along Kundun route, both curves have the same value of electric filed intensity for the first $5 \mathrm{~km}$ but thereafter the experiment curve became consistently higher than the predicted curve by $5.0 \mathrm{mV} / \mathrm{m}$, $1.6 \mathrm{mV} / \mathrm{m}$ and $0.25 \mathrm{mV} / \mathrm{m}$ at $30 \mathrm{~km}, 55 \mathrm{~km}$ and $80 \mathrm{~km}$ respectively as shown in Figure 8 . The path profile is smooth for the wave to follow its contour. Along Fulani route as shown in Figure 9, experimental curve is higher than predicted curve except at $46 \mathrm{~km}$ where they have the same value of electric filed intensity. Experimental curve is higher by $6.0 \mathrm{mV} /$ $\mathrm{m}, 1.0 \mathrm{mV} / \mathrm{m}$ and $0.3 \mathrm{mV} / \mathrm{m}$ at $20 \mathrm{~km}, 47 \mathrm{~km}$ and $54 \mathrm{~km}$ respectively. The path profile is the best among the routes.

Along Dutse Kan route, experimental curve is higher than predicted curve except at $45 \mathrm{~km}$ and thereafter the predicted curve became higher even though the experimental curve attempted but the value is not significant. As shown in Figure 10, the experimental curve is higher by $3.0 \mathrm{mV} / \mathrm{m}$ and $4.0 \mathrm{mV} / \mathrm{m}$ at $18 \mathrm{~km}$ and $35 \mathrm{~km}$ respectively while predicted curve is higher by $0.2 \mathrm{mV} / \mathrm{m}$ and $0.1 \mathrm{mV} / \mathrm{m}$ at $51 \mathrm{~km}$ and $72 \mathrm{~km}$ respectively. The path profile is considerably smooth.

\section{CONCLUSION}

This study investigates the variation in electric field intensity of MF Radio station located at Dutse Jigawa State and compares the adequacy and suitability of predicted propagation curve to characterize MF propagation in Northern Nigeria.

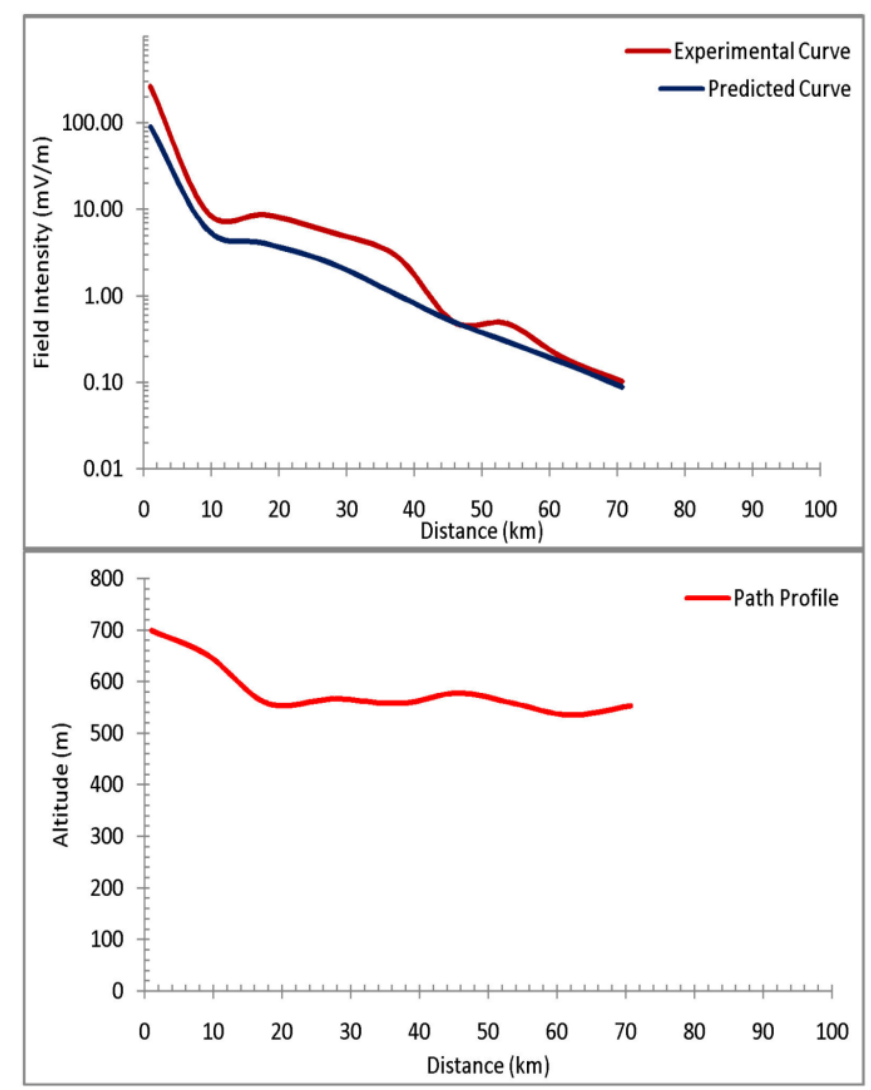

Figure 9: Propagation Curve and Terrain Profiles along Transmitter - Fulani Route

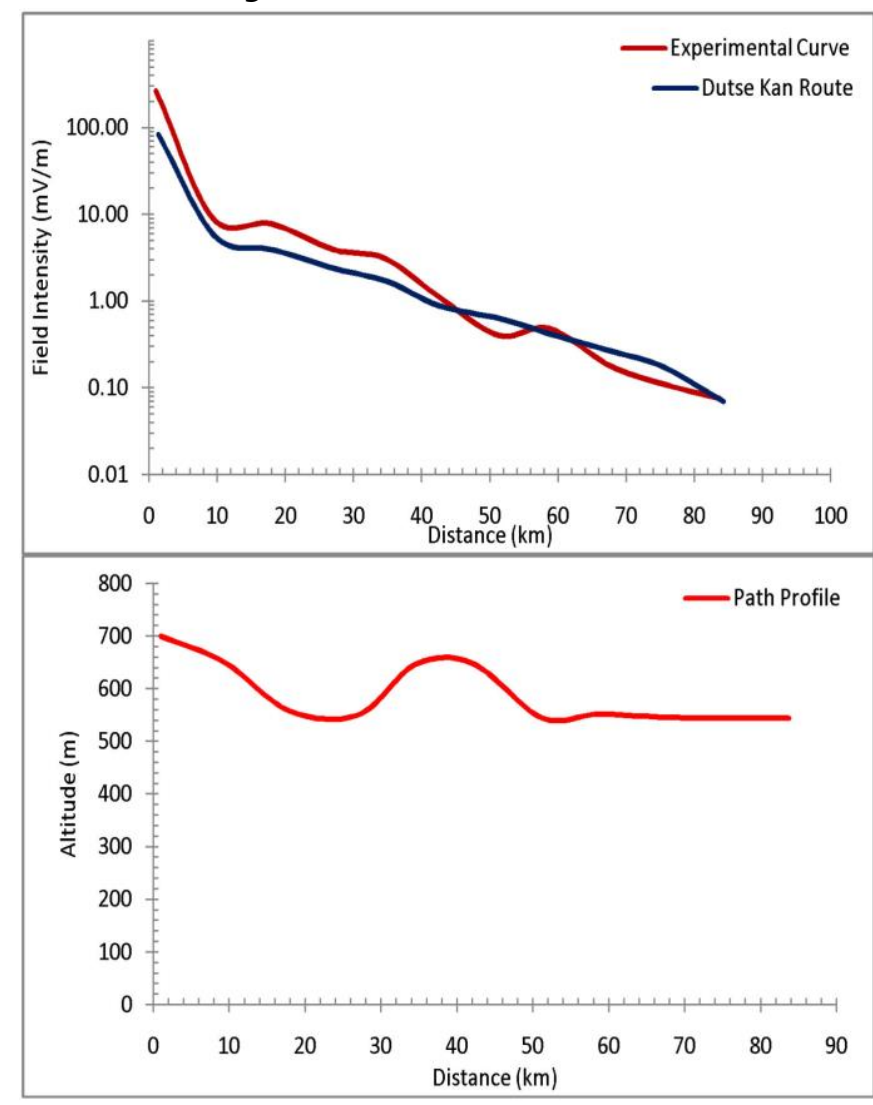

Figure 10: Propagation Curve and Terrain Profiles along Transmitter - Dutse Kan Route 
The results reveal that variation in curves is moderate except at about $10 \mathrm{~km}$ where there is a sharp drop in electric field intensity. It also shows that predicted curve can adequately be used to characterize MF propagation with very limited error because the experimental curves are fairly and generally greater than the predicted curves. The propagation curves produced by $[13,15,20,22]$ assumed the electrical characteristics of the terrain are uniform over the whole routes while this study considered radio routes with mixed electrical characteristics.

\section{REFERENCES}

[1] Seybold S. J. (2005). Introduction to RF Propagation. John Willey and Sons Inc. Canada.

[2] Adenodi R. A., Ajewole M. O., Ogolo, E. O. and Adediji, A. T. (2013a). Application of Amplitude Modulated Medium Wave Transmitters in Deploying Advanced Traveller Information System Australian Journal of Basic and Applied Sciences, 7 (7): 35-42.

[3] ITU-R, Recommendation P. 1321 (1997). Propagation Factors Affecting Systems using Digital Modulation Techniques at LF and MF, pp $1-8$.

[4] International Telecommunication Union, Recommendation ITU-R, P.1321-2 (2007). Propagation Factors Affecting Systems using Digital Modulation Techniques at LF and MF, pp 1-8.

[5] Makki S. V. and Ershadi T. Z. (2008). Determining the Specific Ground Conductivity Aided by the Horizontal Electric Dipole Antenna Near the Ground Surface. World Applied Sciences Journal 3 (1), 122-131.

[6] Marcos, C. G. (2009). Calculation of Radio Electrical Coverage in Medium-Wave Frequencies. EVIC, pp 9 - 10.

[7] European Radio communications Committee Report 77 (2000). Field Strength Measurements along Route. Naples, Japan.

[8] International Telecommunication Union, Recommendation ITU-R SM. 1708 (2005). Field Strength Measurements along a Route with Geographical Coordinate Registrations.

[9] Morris S. and William O. (1973). Essential of Communication Electronics. Peter Peregrinus Ltd., United Kingdon, pp $160-161$.

[10] Ajewole M. O., Oyedum O. D., Adediji A. T., Eichie J. O. and Moses A. S. (2012). "Spatial Coverage of FM Radio Transmitters in Niger State, Nigeria." The IUP Journal of Telecommunications, IV (4): $7-20$.

[11]DeMinco N. (2000). "Propagation prediction techniques and antenna modeling (150 to 1705
$\mathrm{kHz}$ ) for Intelligent Transportation Systems (ITS) broadcast applications," IEEE Antennas and Propagation Magazine, vol. 42, no. 4, pp. 9-34.

[12] Makki S. V., Erhadi T. Z. and Pahlavani F. (2008). Assessment of the Impacts due to the Alternation of Ground Conductivity over the Earth wave field Intensity in MW Band. Progress in Electromagnetics Research B, 4, pp 183 - 195.

[13] Ajayi G. O. and Owolabi I. E. (1975). Propagation Curves (for use in medium wave transmission planning and design). Department of Electronics and Electrical Engineering, University of Ife, Nigeria, pp 1 - 12.

[14] Akinbolati A., Akinsanmi O. and Ekundayo K. R. (2016). Signal Strength Variation and Propagation Profiles of UHF Radio Wave Channel in Ondo State, Nigeria. I.J. Wireless and Microwave Technologies, 4, 12-28.

[15] International Telecommunication Union, Recommendation ITU-R, P-368-7, (1992). "Ground wave propagation curves for frequencies between $10 \mathrm{kHz}$ and $30 \mathrm{MHz}$ ", International Telecommunications Union, pp 1 - 3.

[16] Zeinab T., Hamide K., Mesound B., Mehri M. and Javad, A. S. (2013). Received Signal Strength Estimation in Vehicle-to-Vehicle Communications Using Neural Networks, International Journal of Digital Information and Wireless Communications Vol. 3 (3), 26-30.

[17] Ajewole M. O. and Arogunjo A. M. (2000). Measurement of Ground Electrical Conductivity for Panning Medium Wave Radio Broadcast Stations in South Western Nigeria. Nigeria Journal of Pure and Applied Physics, Vol.1 pp1116.

[18] Deng H. W., Zhao Y. J., Liang C. J., Jiang W. S. and Ning Y. M. (2009). Effective Skin Depth for Multilayer Coated Conductor. Progress in Electromagnetics Research M, Vol. 9, 1-8.

[19] Meng Y. S, Lee Y. H. and Hg B. C. (2009). Study of Propagation Loss in Forest Environment. Progress in Electromagnetics Research B, Vol. 17, $117-133$.

[20] ITU-BR, Recommendation 368-2 (1974): Ground Wave Propagation Curves for frequencies between $10 \mathrm{KHZ}$ and $10 \mathrm{MHz}$, pp.

[21] North Atlantic Treaty Organisation and Research and Technical Organisation (2007).H F Interference, Procedures and Tools. Final Report of NATO RTO Information Systems Technology (IST) Panel Research Task Group IST-050/RTG022.

[22] International Telecommunication Union, Recommendation ITU-R, P-368-9, (2007) "Ground wave propagation curves for frequencies between $10 \mathrm{kHz}$ and $30 \mathrm{MHz}$ ", International Telecommunications Union, pp 1 -5. 